\title{
On toric generators in the unitary and special unitary bordism rings
}

\author{
ZHI LÜ \\ TARAS PANOV
}

\begin{abstract}
We construct a new family of toric manifolds generating the unitary bordism ring. Each manifold in the family is the complex projectivisation of the sum of a line bundle and a trivial bundle over a complex projective space. We also construct a family of special unitary quasitoric manifolds which contains polynomial generators of the special unitary bordism ring with 2 inverted in dimensions $>8$. Each manifold in the latter family is obtained from an iterated complex projectivisation of a sum of line bundles by amending the complex structure to make the first Chern class vanish.
\end{abstract}

57R77; 14M25

\section{Introduction}

Finding geometric representatives of bordism classes is a classical problem on the borders of geometry and topology. The theory of bordism and cobordism is one of the deepest and most influential parts of algebraic topology, which experienced a spectacular development in the 1960s. Although the original definition of bordism, going back to Pontryagin and Thom, was very geometric, it soon became clear that elaborate homotopy-theoretic, algebraic and number-theoretic techniques were required to obtain structural results on bordism groups and (co)bordism rings.

Most calculations of bordism rings of a point for the classical series of Lie groups were settled by coordinated efforts of many topologists by the end of the 1960s (with the notable exception of symplectic bordism, whose structure is still not described completely). These results were summarised in the monograph by Stong [16]. Nevertheless, it has remained a challenging task to describe particular geometric representatives for generators of bordism rings (which tend to be rings of polynomials when 2 is inverted) and other "special" bordism classes. The importance of this problem was much emphasised in the original works such as Conner and Floyd [7].

Over the rationals, the bordism rings are generated by projective spaces, but the integral generators are more subtle as they involve divisibility conditions on characteristic 
numbers. One of the few general results on geometric representatives for bordism classes known from the early 1960s is that the complex bordism ring $\Omega^{\mathrm{U}}$, which is an integral polynomial ring, can be generated by the so-called Milnor hypersurfaces $H\left(n_{1}, n_{2}\right)$. These are hyperplane sections of the Segre embeddings of products $\mathbb{C} P^{n_{1}} \times \mathbb{C} P^{n_{2}}$ of complex projective spaces. Similar generators exist for unoriented and oriented bordism rings.

The early progress was impeded by the lack of examples of higher-dimensional (stably) complex manifolds for which the characteristic numbers can be calculated explicitly. With the appearance of toric varieties in the late 1970s and subsequent development of toric topology (see Buchstaber and Panov [2]), a host of concrete examples of complex manifolds with large symmetry groups has been produced for which characteristic numbers can be calculated effectively using combinatorial-geometric techniques.

In [5], Buchstaber and Ray constructed a set of generators for $\Omega^{\mathrm{U}}$ consisting entirely of complex projective toric manifolds $B\left(n_{1}, n_{2}\right)$, which are projectivisations of sums of line bundles over bounded flag manifolds. Later it was shown in Buchstaber, Panov and Ray [3] that one can get a geometric representative in every complex bordism class if toric manifolds are relaxed to quasitoric ones, the latter still have a "large torus" action, but are only stably complex instead of being complex. Characteristic numbers of toric manifolds satisfy quite restrictive conditions (eg their Todd genus is always 1) which prevent the existence of a toric representative in every bordism class; quasitoric manifolds enjoy more flexibility. We note that representing polynomial generators of $\Omega^{\mathrm{U}}$ by toric manifolds remains open; some progress has been made by Wilfong[17].

Here we consider a family of projective toric manifolds obtained by iterated projectivisation of sums of line bundles, starting from a complex projective space. Such iterated projectivisations are also known as generalised Bott manifolds (see Masuda and Suh [12], and Buchstaber and Panov [2, Section 7.8]). Our first result (Theorem 3.8) shows that the complex bordism ring $\Omega^{\mathrm{U}}$ can be generated by the most simple nontrivial two-stage projectivisations: manifolds $L\left(n_{1}, n_{2}\right)=\mathbb{C} P(\xi)$, where $\xi$ is the sum of a tautological line bundle and an $n_{2}$-dimensional trivial bundle over $\mathbb{C} P^{n_{1}}$. This new toric generator set is somewhat simpler than either of the set of Milnor hypersurfaces $\left\{H\left(n_{1}, n_{2}\right)\right\}$ or Buchstaber and Ray's toric set $\left\{B\left(n_{1}, n_{2}\right)\right\}$.

We proceed by providing explicit families of quasitoric SU-manifolds which contain polynomial generators of the SU-bordism ring $\Omega^{\mathrm{SU}} \otimes \mathbb{Z}\left[\frac{1}{2}\right]$ (Theorem 4.19). In fact, our quasitoric SU-manifolds are genuinely indecomposable and indivisible elements in $\Omega^{\mathrm{SU}}$ (integrally, without inverting any prime), however $\Omega^{\mathrm{SU}}$ is not a polynomial ring.

We recall that a stably complex (or unitary) manifold $M$ is special unitary (an SU-manifold for short) if $c_{1}(M)=0$. A renewed interest to this class of manifolds 
has been stimulated by the development of geometry motivated by physics; the notion of a Calabi-Yau manifold plays a central role here. By a Calabi-Yau manifold one usually understands a Kähler SU-manifold; it has a Ricci flat metric by the theorem of Yau. We note however that our SU-manifolds are rarely Kähler.

As was observed by Lü and Wang in [11], quasitoric SU-manifolds can be constructed by taking iterated complex projectivisations (which are projective toric manifolds) and then amending the stably complex structure so that the first Chern class becomes zero. The underlying smooth manifold of the result is still toric, but the stably complex structure is not the standard one. Examples of this sort were known to Conner and Floyd and used in their constructions [7], however the existence of a torus action was not emphasised and their amended stably complex structures were actually not SU.

The characteristic numbers of SU-manifolds satisfy intricate divisibility conditions. Ochanine's theorem [14] asserting that the signature of an $(8 k+4)$-dimensional $\mathrm{SU}-$ manifold is divisible by 16 is one of the most famous examples. We therefore find it quite miraculous that polynomial generators for the SU-bordism ring $\Omega^{\mathrm{SU}}$ occur within the most basic families of examples that one can produce using toric methods: two-stage complex projectivisations, and three-stage projectivisations with the first stage being just $\mathbb{C} P^{1}$. The proof of Theorem 4.19 involves calculating the characteristic numbers and checking various divisibility conditions. We use both classical and more recent results on binomial coefficients modulo a prime.

We note also that the existence of large torus actions indicates possible applications of our examples in the equivariant setting. Applicability of toric methods in equivariant bordism is currently being explored (see Buchstaber, Panov and Ray [4], Buchstaber and Panov [2, Chapter 9], and Lü [10]).

Acknowledgements We thank Peter Landweber and the referee for their most helpful comments. Lü was supported by the NSFC, grants 11371093, 11431009 and 11661131004. Panov was supported by the Russian Foundation for Basic Research (grants 14-01-00537 and 16-51-55017) and a grant from Dmitri Zimin's "Dynasty" foundation. Both authors were supported by the Key Laboratory of Mathematics for Nonlinear Sciences in Fudan University, Chinese Ministry of Education.

\section{Toric and quasitoric manifolds, cohomology and Chern classes}

Here we collect the necessary information about toric varieties and quasitoric manifolds; the details can be found in [2]. 
A toric variety is a normal complex algebraic variety $V$ containing an algebraic torus $\left(\mathbb{C}^{\times}\right)^{n}$ as a Zariski open subset in such a way that the natural action of $\left(\mathbb{C}^{\times}\right)^{n}$ on itself extends to an action on $V$. We only consider nonsingular complete (compact in the usual topology) toric varieties, also known as toric manifolds.

There is a bijective correspondence between the isomorphism classes of complex $n$-dimensional toric manifolds and complete regular fans in $\mathbb{R}^{n}$. A fan is a finite collection $\Sigma=\left\{\sigma_{1}, \ldots, \sigma_{s}\right\}$ of strongly convex cones $\sigma_{i}$ in $\mathbb{R}^{n}$ such that every face of a cone in $\Sigma$ belongs to $\Sigma$ and the intersection of any two cones in $\Sigma$ is a face of each. A fan $\Sigma$ is regular if each of its cones $\sigma_{j}$ is generated by part of a basis of the lattice $\mathbb{Z}^{n} \subset \mathbb{R}^{n}$ (we choose the standard lattice for simplicity). In particular, each 1 -dimensional cone of $\Sigma$ is generated by a primitive vector $\boldsymbol{a}_{i} \in \mathbb{Z}^{n}$. A fan $\Sigma$ is complete if the union of its cones is the whole $\mathbb{R}^{n}$.

Projective toric varieties are particularly important. A projective toric manifold $V$ is defined by a lattice Delzant polytope $P$. Given a simple $n$-dimensional polytope $P$ with vertices in the lattice $\mathbb{Z}^{n}$, one defines the normal fan $\Sigma_{P}$ as the fan whose $n$-dimensional cones $\sigma_{v}$ correspond to the vertices $v$ of $P$, and $\sigma_{v}$ is generated by the primitive inside-pointing normals to the facets of $P$ meeting at $v$. The polytope $P$ is Delzant precisely when its normal fan $\Sigma_{P}$ is regular. The fan $\Sigma_{P}$ defines a projective toric manifold $V_{P}$. Different lattice Delzant polytopes with the same normal fan produce different projective embeddings of the same toric manifold.

Irreducible torus-invariant divisors on $V$ are the toric subvarieties of complex codimension 1 corresponding to the 1 -dimensional cones of $\Sigma$. When $V$ is projective, they also correspond to the facets of $P$. We assume that there are $m 1$-dimensional cones (or facets), denote the corresponding primitive vectors by $\boldsymbol{a}_{1}, \ldots, \boldsymbol{a}_{m}$, and denote the corresponding codimension- 1 subvarieties by $V_{1}, \ldots, V_{m}$.

Theorem 2.1 Let $V$ be a toric manifold of complex dimension $n$, with the corresponding complete regular fan $\Sigma$. The cohomology ring $H^{*}(V ; \mathbb{Z})$ is generated by the degree-two classes $v_{i}$ dual to the invariant submanifolds $V_{i}$, and is given by

$$
H^{*}(V ; \mathbb{Z}) \cong \mathbb{Z}\left[v_{1}, \ldots, v_{m}\right] / \mathcal{I}, \quad \operatorname{deg} v_{i}=2,
$$

where $\mathcal{I}$ is the ideal generated by elements of the following two types:

(a) $v_{i_{1}} \cdots v_{i_{k}}$ such that $\boldsymbol{a}_{i_{1}}, \ldots, \boldsymbol{a}_{i_{k}}$ do not span a cone of $\Sigma$;

(b) $\sum_{i=1}^{m}\left\langle\boldsymbol{a}_{i}, \boldsymbol{x}\right\rangle v_{i}$, for any vector $\boldsymbol{x} \in \mathbb{Z}^{n}$.

It is convenient to consider the integer $n \times m$-matrix

$$
\Lambda=\left(\begin{array}{ccc}
a_{11} & \cdots & a_{1 m} \\
\vdots & \ddots & \vdots \\
a_{n 1} & \cdots & a_{n m}
\end{array}\right)
$$


whose columns are the vectors $\boldsymbol{a}_{i}$ written in the standard basis of $\mathbb{Z}^{n}$. Then the ideal (b) of Theorem 2.1 is generated by the $n$ linear forms $a_{j 1} v_{1}+\cdots+a_{j m} v_{m}$ corresponding to the rows of $\Lambda$.

Theorem 2.2 There is the following isomorphism of complex vector bundles:

$$
\mathcal{T} V \oplus \underline{\mathbb{C}}^{m-n} \cong \rho_{1} \oplus \cdots \oplus \rho_{m},
$$

where $\mathcal{T} V$ is the tangent bundle, $\underline{\mathbb{C}}^{m-n}$ is the trivial $(m-n)$-plane bundle, and $\rho_{i}$ is the line bundle corresponding to $V_{i}$, with $c_{1}\left(\rho_{i}\right)=v_{i}$. In particular, the total Chern class of $V$ is given by

$$
c(V)=\left(1+v_{1}\right) \cdots\left(1+v_{m}\right) .
$$

Example 2.3 A basic example of a toric manifold is the complex projective space $\mathbb{C} P^{n}$. The cones of the corresponding fan are generated by proper subsets of the set of $m=n+1$ vectors $\boldsymbol{e}_{1}, \ldots, \boldsymbol{e}_{n},-\boldsymbol{e}_{1}-\cdots-\boldsymbol{e}_{n}$, where $\boldsymbol{e}_{i} \in \mathbb{Z}^{n}$ is the $i^{\text {th }}$ standard basis vector. It is the normal fan of the lattice simplex $\Delta^{n}$ with the vertices at $\mathbf{0}$ and $\boldsymbol{e}_{1}, \ldots, \boldsymbol{e}_{n}$. The matrix (2-1) is given by

$$
\left(\begin{array}{cccc}
1 & 0 & 0 & -1 \\
0 & \ddots & 0 & \vdots \\
0 & 0 & 1 & -1
\end{array}\right) .
$$

Theorem 2.1 gives the cohomology of $\mathbb{C} P^{n}$ as

$H^{*}\left(\mathbb{C} P^{n}\right) \cong \mathbb{Z}\left[v_{1}, \ldots, v_{n+1}\right] /\left(v_{1} \cdots v_{n+1}, v_{1}-v_{n+1}, \ldots, v_{n}-v_{n+1}\right) \cong \mathbb{Z}[v] /\left(v^{n+1}\right)$,

where $v$ is any of the $v_{i}$. Theorem 2.2 gives the standard decomposition

$$
\mathcal{T} \mathbb{C} P^{n} \oplus \underline{\mathbb{C}} \cong \bar{\eta} \oplus \cdots \oplus \bar{\eta} \quad(n+1 \text { summands }),
$$

where $\eta=\mathcal{O}(-1)$ is the tautological (Hopf) line bundle over $\mathbb{C} P^{n}$, and $\bar{\eta}=\mathcal{O}(1)$ is its conjugate, or the line bundle corresponding to a hyperplane $\mathbb{C} P^{n-1} \subset \mathbb{C} P^{n}$.

Example 2.4 An example which will be important for our constructions is the complex projectivisation of a sum of line bundles over projective space.

Given two positive integers $n_{1}, n_{2}$ and a sequence of integers $\left(i_{1}, \ldots, i_{n_{2}}\right)$, consider the projectivisation $V=\mathbb{C} P\left(\eta^{\otimes i_{1}} \oplus \cdots \oplus \eta^{\otimes i_{n_{2}}} \oplus \mathbb{C}\right)$, where $\eta^{\otimes i}$ denotes the $i^{\text {th }}$ tensor power of $\eta$ over $\mathbb{C} P^{n_{1}}$ when $i \geqslant 0$ and the $i^{\text {th }}$ tensor power of $\bar{\eta}$ otherwise. The manifold $V$ is the total space of a bundle over $\mathbb{C} P^{n_{1}}$ with fibre $\mathbb{C} P^{n_{2}}$. It is also a projective toric manifold with the corresponding matrix (2-1) given by 


$$
\left(\begin{array}{cccccccc}
\overbrace{1}^{n_{1}} & 0 & 0 & -1 & & & \\
0 & \ddots & 0 & \vdots & & 0 & \\
0 & 0 & 1 & -1 & & & \\
& & & i_{1} & 1 & 0 & 0 & -1 \\
& 0 & & \vdots & 0 & \ddots & 0 & \vdots \\
& & & i_{n_{2}} & \underbrace{0}_{n_{2}} & 0 & 1 & -1
\end{array}\right) .
$$

The polytope $P$ here is combinatorially equivalent to a product $\Delta^{n_{1}} \times \Delta^{n_{2}}$ of two simplices. Theorem 2.1 gives the cohomology of $V$ as

$$
H^{*}(V) \cong \mathbb{Z}\left[v_{1}, \ldots, v_{n_{1}+1}, v_{n_{1}+2}, \ldots, v_{n_{1}+n_{2}+2}\right] / \mathcal{I},
$$

where $\mathcal{I}$ is generated by the elements

$$
\begin{aligned}
& v_{1} \cdots v_{n_{1}+1}, \quad v_{n_{1}+2} \cdots v_{n_{1}+n_{2}+2}, \quad v_{1}-v_{n_{1}+1}, \quad \ldots, \quad v_{n_{1}}-v_{n_{1}+1}, \\
& i_{1} v_{n_{1}+1}+v_{n_{1}+2}-v_{n_{1}+n_{2}+2}, \quad \ldots, \quad i_{n_{2}} v_{n_{1}+1}+v_{n_{1}+n_{2}+1}-v_{n_{1}+n_{2}+2} \text {. }
\end{aligned}
$$

In other words,

$$
H^{*}(V) \cong \mathbb{Z}[u, v] /\left(u^{n_{1}+1}, v\left(v-i_{1} u\right) \cdots\left(v-i_{n_{2}} u\right)\right),
$$

where $u=v_{1}=\cdots=v_{n_{1}+1}$ and $v=v_{n_{1}+n_{2}+2}$. Theorem 2.2 gives

$$
c(V)=(1+u)^{n_{1}+1}\left(1+v-i_{1} u\right) \cdots\left(1+v-i_{n_{2}} u\right)(1+v) .
$$

If $i_{1}=\cdots=i_{n_{2}}=0$, we obtain $V=\mathbb{C} P^{n_{1}} \times \mathbb{C} P^{n_{2}}$.

The same information can be retrieved from the following well-known description of the tangent bundle and the cohomology ring of a complex projectivisation:

Theorem 2.5 (Borel and Hirzebruch [1, Section 15]) Let $p: \mathbb{C} P(\xi) \rightarrow X$ be the projectivisation of a complex $n$-plane bundle $\xi$ over a complex manifold $X$, and let $\gamma$ be the tautological line bundle over $\mathbb{C P}(\xi)$. Then there is an isomorphism of vector bundles

$$
\mathcal{T} \mathbb{C} P(\xi) \oplus \underline{\mathbb{C}} \cong p^{*} \mathcal{T} X \oplus\left(\bar{\gamma} \otimes p^{*} \xi\right),
$$

where $\mathbb{C}$ denotes a trivial line bundle over $\mathbb{C} P(\xi)$. Furthermore, the integral cohomology ring of $\mathbb{C} P(\xi)$ is the quotient of the polynomial ring $H^{*}(X)[v]$ on one generator $v=c_{1}(\bar{\gamma})$ with coefficients in $H^{*}(X)$ by the single relation

$$
v^{n}+c_{1}(\xi) v^{n-1}+\cdots+c_{n}(\xi)=0 .
$$


The relation above is just $c_{n}\left(\bar{\gamma} \otimes p^{*} \xi\right)=0$.

In the case considered above, $\xi=\eta^{\otimes i_{1}} \oplus \cdots \oplus \eta^{\otimes i_{n_{2}}} \oplus \underline{\mathbb{C}}$ over $X=\mathbb{C} P^{n_{1}}$. We then have $H^{*}(X)=\mathbb{Z}[u] /\left(u^{n_{1}+1}\right)$ where $u=c_{1}(\bar{\eta})$, so that equation (2-4) becomes $v(v-$ $\left.i_{1} u\right) \cdots\left(v-i_{n_{2}} u\right)=0$ and the ring $H^{*}(\mathbb{C} P(\xi))$ given by Theorem 2.5 is precisely (2-2). Further, the total Chern class of $p^{*} \mathcal{T} X \oplus\left(\bar{\gamma} \otimes p^{*} \xi\right)$ is given by (2-3).

The quotient of the projective toric manifold $V_{P}$ by the action of the compact torus $T^{n} \subset\left(\mathbb{C}^{\times}\right)^{n}$ is the polytope $P$.

A quasitoric manifold over a combinatorial simple $n$-dimensional polytope $P$ is a manifold $M$ of dimension $2 n$ with a locally standard action of $T^{n}$ such that the quotient $M / T^{n}$ is homeomorphic, as a manifold with corners, to $P$. (An action of $T^{n}$ on $M^{2 n}$ is locally standard if every point $x \in M^{2 n}$ is contained in a $T^{n}$-invariant neighbourhood equivariantly homeomorphic to an open subset in $\mathbb{C}^{n}$ with the standard coordinatewise action of $T^{n}$ twisted by an automorphism of the torus; the orbit space of a locally standard action is a manifold with corners.) We therefore have a projection $\pi: M \rightarrow P$ whose fibres are orbits of the $T^{n}$-action.

Not every simple polytope can be the quotient of a quasitoric manifold. Nevertheless, quasitoric manifolds constitute a much larger family than projective toric manifolds, and enjoy more flexibility for topological applications.

If $F_{1}, \ldots, F_{m}$ are facets of $P$, then each $M_{i}=\pi^{-1}\left(F_{i}\right)$ is a quasitoric submanifold of $M$ of codimension 2, called a characteristic submanifold. The characteristic submanifolds $M_{i} \subset M$ are analogues of the invariant divisors $V_{i}$ on a toric manifold $V$. Each $M_{i}$ is fixed pointwise by a closed 1-dimensional subgroup (a subcircle) $T_{i} \subset T^{n}$ and therefore corresponds to a primitive vector $\lambda_{i} \in \mathbb{Z}^{n}$ defined up to a sign. Choosing a direction of $\lambda_{i}$ is equivalent to choosing an orientation for the normal bundle $v\left(M_{i} \subset M\right)$ or, equivalently, choosing an orientation for $M_{i}$, provided that $M$ itself is oriented. An omniorientation of a quasitoric manifold $M$ consists of a choice of orientation for $M$ and each characteristic submanifold $M_{i}, 1 \leqslant i \leqslant m$.

The vectors $\lambda_{i}$ are analogues of the generators $\boldsymbol{a}_{i}$ of the 1-dimensional cones of the fan corresponding to a toric manifold $V$ (or analogues of the normal vectors to the facets of $P$ when $V$ is projective). However, the $\lambda_{i}$ need not be the normal vectors to the facets of $P$ in general.

There is an analogue of Theorem 2.1 for quasitoric manifolds:

Theorem 2.6 Let $M$ be an omnioriented quasitoric manifold of dimension $2 n$ over a polytope $P$. The cohomology ring $H^{*}(M ; \mathbb{Z})$ is generated by the degree-two 
classes $v_{i}$ dual to the oriented characteristic submanifolds $M_{i}$, and is given by

$$
H^{*}(M ; \mathbb{Z}) \cong \mathbb{Z}\left[v_{1}, \ldots, v_{m}\right] / \mathcal{I}, \quad \operatorname{deg} v_{i}=2,
$$

where $\mathcal{I}$ is the ideal generated by elements of the following two types:

(a) $v_{i_{1}} \cdots v_{i_{k}}$ such that $F_{i_{1}} \cap \cdots \cap F_{i_{k}}=\varnothing$ in $P$;

(b) $\sum_{i=1}^{m}\left\langle\lambda_{i}, \boldsymbol{x}\right\rangle v_{i}$, for any vector $\boldsymbol{x} \in \mathbb{Z}^{n}$.

By analogy with (2-1), we consider the integer $n \times m$-matrix

$$
\Lambda=\left(\begin{array}{ccc}
\lambda_{11} & \cdots & \lambda_{1 m} \\
\vdots & \ddots & \vdots \\
\lambda_{n 1} & \cdots & \lambda_{n m}
\end{array}\right)
$$

whose columns are the vectors $\lambda_{i}$ written in the standard basis of $\mathbb{Z}^{n}$. Changing a basis in the lattice results in multiplying $\Lambda$ from the left by a matrix from $\operatorname{GL}(n, \mathbb{Z})$. The ideal (b) of Theorem 2.6 is generated by the $n$ linear forms $\lambda_{j 1} v_{1}+\cdots+\lambda_{j m} v_{m}$ corresponding to the rows of $\Lambda$. Also, $\Lambda$ has the property that $\operatorname{det}\left(\lambda_{i_{1}}, \ldots, \lambda_{i_{n}}\right)= \pm 1$ whenever the facets $F_{i_{1}}, \ldots, F_{i_{n}}$ intersect at a vertex of $P$.

There is also an analogue of Theorem 2.2:

Theorem 2.7 For a quasitoric manifold $M$ of dimension $2 n$, there is an isomorphism of real vector bundles:

$$
\mathcal{T} M \oplus \underline{\mathbb{R}}^{2(m-n)} \cong \rho_{1} \oplus \cdots \oplus \rho_{m},
$$

where $\rho_{i}$ is the real 2-plane bundle corresponding to the orientable characteristic submanifold $M_{i} \subset M$, so that $\left.\rho_{i}\right|_{M_{i}}=v\left(M_{i} \subset M\right)$.

\section{Unitary bordism}

Here we provide a new set of toric generators for the unitary bordism ring. The general information about unitary (or complex) bordism can be found in [16].

Elements of the unitary bordism ring $\Omega^{\mathrm{U}}$ are the complex bordism classes of stably complex manifolds. A stably complex manifold is a pair $\left(M, c_{\mathcal{T}}\right)$ consisting of a smooth manifold $M$ and a stably complex structure $c_{\mathcal{T}}$, where the latter is determined by a choice of an isomorphism

$$
c_{\mathcal{T}}: \mathcal{T} M \oplus \underline{\mathbb{R}}^{N} \stackrel{\cong}{\longrightarrow} \xi
$$


between the stable tangent bundle of $M$ and a complex vector bundle $\xi$. We omit $c_{\mathcal{T}}$ in the notation when it is clear from the context. We denote by $[M] \in \Omega^{\mathrm{U}}$ the bordism class of a stably complex manifold $M$. The sum in $\Omega^{\mathrm{U}}$ is the disjoint union, and the product is induced by the Cartesian product of manifolds. The ring $\Omega^{\mathrm{U}}$ is graded by the dimension of manifolds.

A complex manifold $M$ (in particular, a toric manifold) has a canonical stably complex structure arising from the complex structure on $\mathcal{T} M$. An omniorientation of a quasitoric manifold $M$ gives it a stably complex structure by means of the isomorphism of Theorem 2.7, because a choice of orientation for each real 2-plane bundle $\rho_{i}$ is equivalent to endowing it with a complex structure.

Example 3.1 The canonical stably complex structure on $\mathbb{C} P^{n}$ (as a complex manifold) is given by the isomorphism

$$
\mathcal{T} \mathbb{C} P^{n} \oplus \underline{\mathbb{R}}^{2} \cong \bar{\eta} \oplus \cdots \oplus \bar{\eta} \quad(n+1 \text { summands }) .
$$

On the other hand, $\mathbb{C} P^{n}$, viewed as a quasitoric manifold over $\Delta^{n}$, has $n+1$ characteristic submanifolds, and therefore $2^{n+2}$ different omniorientations. Each of these omniorientations gives rise to a stably complex structure, obtained by replacing some of the line bundles $\bar{\eta}$ above with $\eta$, or by reversing the global orientation. Some of these stably complex structures are equivalent, of course.

We have $H^{*}(B U(n)) \cong \mathbb{Z}\left[c_{1}, \ldots, c_{n}\right], \operatorname{deg} c_{i}=2 i$, where the $c_{i}$ are the universal Chern characteristic classes. For any sequence $\omega=\left(i_{1}, \ldots, i_{n}\right)$ of nonnegative integers, there is the monomial $c_{\omega}=c_{1}^{i_{1}} \cdots c_{n}^{i_{n}}$ of degree $2\|\omega\|=2 \sum_{k=1}^{n} k i_{k}$ and the corresponding characteristic class $c_{\omega}(\xi)$ of a complex $n$-plane bundle $\xi$. The corresponding tangential Chern characteristic number of a stably complex manifold $M$ is defined by $c_{\omega}[M]=$ $c_{\omega}(\mathcal{T} M)\langle M\rangle$. Here $\langle M\rangle$ is the fundamental homology class of $M$, and $\mathcal{T} M$ is regarded as a complex bundle via the isomorphism (3-1). The number $c_{\omega}[M]$ is assumed to be zero when $2\|\omega\| \neq \operatorname{dim} M$.

Theorem 3.2 Two stably complex manifold $M$ and $N$ represent the same bordism classes in $\Omega^{\mathrm{U}}$ if and only if their sets of Chern characteristic numbers coincide.

Another important characteristic class is $s_{n}$. It is defined as the polynomial in $c_{1}, \ldots, c_{n}$ obtained by expressing the symmetric polynomial $x_{1}^{n}+\cdots+x_{n}^{n}$ via the elementary symmetric functions $\sigma_{i}\left(x_{1}, \ldots, x_{n}\right)$ and then replacing each $\sigma_{i}$ by $c_{i}$. Define the corresponding characteristic number as $s_{n}[M]=s_{n}(\mathcal{T} M)\langle M\rangle$.

The ring $\Omega^{\mathrm{U}}$ was described by Milnor and Novikov (see [13], and Stong [16]): 
Theorem 3.3 The ring $\Omega^{\mathrm{U}}$ is a polynomial ring on generators in every even degree:

$$
\Omega^{\mathrm{U}} \cong \mathbb{Z}\left[a_{i}, i>0\right], \quad \operatorname{deg} a_{i}=2 i .
$$

Then the bordism class of a stably complex manifold $M^{2 i}$ may be taken to be the $2 i$-dimensional generator $a_{i}$ if and only if

$$
s_{i}\left[M^{2 i}\right]= \begin{cases} \pm 1 & \text { if } i+1 \neq p^{s} \text { for any prime } p \\ \pm p & \text { if } i+1=p^{s} \text { for some prime } p \text { and integer } s>0\end{cases}
$$

There is no universal description of connected manifolds representing the polynomial generators $a_{n} \in \Omega^{\mathrm{U}}$. However, there are known explicit families of manifolds whose bordism classes generate the whole ring $\Omega^{\mathrm{U}}$.

The classical family of generators for the ring $\Omega^{\mathrm{U}}$ consists of the Milnor hypersurfaces $H\left(n_{1}, n_{2}\right)$. Each $H\left(n_{1}, n_{2}\right)$ is a hyperplane section of the Segre embedding $\mathbb{C} P^{n_{1}} \times \mathbb{C} P^{n_{2}} \rightarrow \mathbb{C} P^{\left(n_{1}+1\right)\left(n_{2}+1\right)-1}$ and may be given explicitly by the equation

$$
z_{0} w_{0}+\cdots+z_{n_{1}} w_{n_{1}}=0
$$

in the homogeneous coordinates $\left[z_{0}: \cdots: z_{n_{1}}\right] \in \mathbb{C} P^{n_{1}}$ and $\left[w_{0}: \cdots: w_{n_{2}}\right] \in \mathbb{C} P^{n_{2}}$, assuming that $n_{1} \leqslant n_{2}$. Also, $H\left(n_{1}, n_{2}\right)$ can be identified with the projectivisation $\mathbb{C} P(\zeta)$ of a certain $n_{2}$-plane bundle over $\mathbb{C} P^{n_{1}}$. The bundle $\zeta$ is not a sum of line bundles when $n_{1}>1$, so $H\left(n_{1}, n_{2}\right)$ is not a toric manifold in this case (see [2, Section 9.1]).

Buchstaber and Ray [5] introduced a family $B\left(n_{1}, n_{2}\right)$ of toric generators of $\Omega^{\mathrm{U}}$. Each $B\left(n_{1}, n_{2}\right)$ is the projectivisation of a sum of $n_{2}$ line bundles over the bounded flag manifold $B F_{n_{1}}$. Then $B\left(n_{1}, n_{2}\right)$ is a toric manifold, because $B F_{n_{1}}$ is toric and the projectivisation of a sum of line bundles over a toric manifold is toric.

We have $H\left(0, n_{2}\right)=B\left(0, n_{2}\right)=\mathbb{C} P^{n_{2}-1}$, so

$$
s_{n_{2}-1}\left[H\left(0, n_{2}\right)\right]=s_{n_{2}-1}\left[B\left(0, n_{2}\right)\right]=n_{2} .
$$

Furthermore,

$$
s_{n_{1}+n_{2}-1}\left[H\left(n_{1}, n_{2}\right)\right]=s_{n_{1}+n_{2}-1}\left[B\left(n_{1}, n_{2}\right)\right]=-\left(\begin{array}{c}
n_{1}+n_{2} \\
n_{1}
\end{array}\right) \quad \text { for } n_{1}>1
$$

see [2, Section 9.1] for the details.

We shall need the following two facts from number theory: 
Theorem 3.4 (Lucas) Let $p$ be a prime, and let

$$
\begin{aligned}
n & =n_{0}+n_{1} p+\cdots+n_{k-1} p^{k-1}+n_{k} p^{k}, \\
m & =m_{0}+m_{1} p+\cdots+m_{k-1} p^{k-1}+m_{k} p^{k}
\end{aligned}
$$

be the base $p$ expansions of positive integers $m$ and $n$. Then

$$
\left(\begin{array}{c}
n \\
m
\end{array}\right) \equiv\left(\begin{array}{c}
n_{0} \\
m_{0}
\end{array}\right)\left(\begin{array}{c}
n_{1} \\
m_{1}
\end{array}\right) \cdots\left(\begin{array}{c}
n_{k} \\
m_{k}
\end{array}\right) \bmod p .
$$

Here the standard convention $\left(\begin{array}{c}m \\ n\end{array}\right)=0$ if $m<n$ is used.

For the proof, see eg [15, Lemma 2.6].

Proposition 3.5 For any integer $n>0$, we have

$$
\operatorname{gcd}\left\{\left(\begin{array}{c}
n \\
i
\end{array}\right), 0<i<n\right\}=\left\{\begin{array}{lll}
1 & \text { if } n \neq p^{s} \quad \text { for any prime } p \\
p & \text { if } n=p^{s} \quad \text { for some prime } p \text { and integer } s>0 .
\end{array}\right.
$$

Proof Assume $n=p^{s}$. Then each $\left(\begin{array}{c}n \\ i\end{array}\right)$ with $0<i<n$ is divisible by $p$. On the other hand, $\left(\begin{array}{c}p^{s} \\ p^{s-1}\end{array}\right)$ is not divisible by $p^{2}$, eg by Kummer's theorem.

Now assume $n \neq p^{s}$. Write the base $p$ expansion

$$
n=n_{0}+n_{1} p+\cdots+n_{k-1} p^{k-1}+n_{k} p^{k},
$$

where we may assume $n_{k}>0$. Take

$$
i=n_{0}+n_{1} p+\cdots+n_{k-1} p^{k-1}+\left(n_{k}-1\right) p^{k} .
$$

Then $i \neq 0$ as otherwise $n=p^{k}$. By Theorem 3.4, $\left(\begin{array}{c}n \\ i\end{array}\right) \equiv n_{k} \not \equiv 0 \bmod p$.

The fact that each of the families $\left\{\left[H\left(n_{1}, n_{2}\right)\right]\right\}$ and $\left\{\left[B\left(n_{1}, n_{2}\right)\right]\right\}$ generates the unitary bordism ring $\Omega^{\mathrm{U}}$ follows from (3-2), Proposition 3.5 and Theorem 3.3.

We proceed to describe another family of toric generators for $\Omega^{\mathrm{U}}$.

Construction 3.6 Given two positive integers $n_{1}$ and $n_{2}$, we define the manifold $L\left(n_{1}, n_{2}\right)$ as the projectivisation $\mathbb{C} P\left(\eta \oplus \underline{\mathbb{C}}^{n_{2}}\right)$, where $\eta$ is the tautological line bundle over $\mathbb{C} P^{n_{1}}$. This $L\left(n_{1}, n_{2}\right)$ is a particular case of manifolds from Example 2.4, so it is a projective toric manifold with the corresponding matrix (2-1) given by 


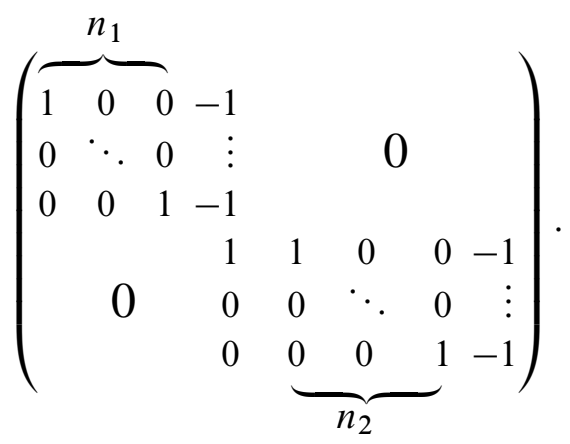

The cohomology ring is given by

$$
H^{*}\left(L\left(n_{1}, n_{2}\right)\right) \cong \mathbb{Z}[u, v] /\left(u^{n_{1}+1}, v^{n_{2}+1}-u v^{n_{2}}\right)
$$

with $u^{n_{1}} v^{n_{2}}\left\langle L\left(n_{1}, n_{2}\right)\right\rangle=1$. There is an isomorphism of complex bundles

$$
\mathcal{T} L\left(n_{1}, n_{2}\right) \oplus \underline{\mathbb{C}}^{2} \cong \underbrace{p^{*} \bar{\eta} \oplus \cdots \oplus p^{*} \bar{\eta}}_{n_{1}+1} \oplus\left(\bar{\gamma} \otimes p^{*} \eta\right) \oplus \underbrace{\bar{\gamma} \oplus \cdots \oplus \bar{\gamma}}_{n_{2}},
$$

where $\gamma$ is the tautological line bundle over $L\left(n_{1}, n_{2}\right)=\mathbb{C} P\left(\eta \oplus \underline{\mathbb{C}}^{n_{2}}\right)$. The total Chern class is

$$
c\left(L\left(n_{1}, n_{2}\right)\right)=(1+u)^{n_{1}+1}(1+v-u)(1+v)^{n_{2}}
$$

with $u=c_{1}\left(p^{*} \bar{\eta}\right)$ and $v=c_{1}(\bar{\gamma})$. We also set $L\left(n_{1}, 0\right)=\mathbb{C} P^{n_{1}}$ and $L\left(0, n_{2}\right)=\mathbb{C} P^{n_{2}}$, then the identities (3-4)-(3-6) still hold.

Lemma 3.7 For $n_{2}>0$, we have

$$
s_{n_{1}+n_{2}}\left[L\left(n_{1}, n_{2}\right)\right]=\left(\begin{array}{c}
n_{1}+n_{2} \\
0
\end{array}\right)-\left(\begin{array}{c}
n_{1}+n_{2} \\
1
\end{array}\right)+\cdots+(-1)^{n_{1}}\left(\begin{array}{c}
n_{1}+n_{2} \\
n_{1}
\end{array}\right)+n_{2} .
$$

Proof Using (3-6) and (3-4) we calculate

$$
\begin{aligned}
s_{n_{1}+n_{2}}\left(L\left(n_{1}, n_{2}\right)\right)= & (v-u)^{n_{1}+n_{2}}+n_{2} v^{n_{1}+n_{2}} \\
= & \left(\begin{array}{c}
n_{1}+n_{2} \\
0
\end{array}\right) v^{n_{1}+n_{2}}-\left(\begin{array}{c}
n_{1}+n_{2} \\
1
\end{array}\right) u v^{n_{1}+n_{2}-1}+\cdots \\
& \quad+(-1)^{n_{1}}\left(\begin{array}{c}
n_{1}+n_{2} \\
n_{1}
\end{array}\right) u^{n_{1}} v^{n_{2}}+n_{2} v^{n_{1}+n_{2}} \\
= & \left(\left(\begin{array}{c}
n_{1}+n_{2} \\
0
\end{array}\right)-\left(\begin{array}{c}
n_{1}+n_{2} \\
1
\end{array}\right)+\cdots+(-1)^{n_{1}}\left(\begin{array}{c}
n_{1}+n_{2} \\
n_{1}
\end{array}\right)+n_{2}\right) u^{n_{1}} v^{n_{2}},
\end{aligned}
$$

and the result follows by evaluating at $\left\langle L\left(n_{1}, n_{2}\right)\right\rangle$.

Theorem 3.8 The bordism classes $\left[L\left(n_{1}, n_{2}\right)\right] \in \Omega_{2\left(n_{1}+n_{2}\right)}^{\mathrm{U}}$ generate the ring $\Omega^{\mathrm{U}}$. 
Proof Assuming $\left[L\left(n_{1}, n_{2}\right)\right]=0$ when $n_{1}<0$, we calculate using Lemma 3.7:

$$
\begin{aligned}
s_{n_{1}+n_{2}}\left[L\left(n_{1}, n_{2}\right)\right. & \left.-2 L\left(n_{1}-1, n_{2}+1\right)+L\left(n_{1}-2, n_{2}+2\right)\right] \\
= & (-1)^{n_{1}-1}\left(\begin{array}{c}
n_{1}+n_{2} \\
n_{1}-1
\end{array}\right)+(-1)^{n_{1}}\left(\begin{array}{c}
n_{1}+n_{2} \\
n_{1}
\end{array}\right)-2(-1)^{n_{1}-1}\left(\begin{array}{c}
n_{1}+n_{2} \\
n_{1}-1
\end{array}\right) \\
= & (-1)^{n_{1}}\left(\begin{array}{c}
n_{1}+n_{2}+1 \\
n_{1}
\end{array}\right) .
\end{aligned}
$$

The result follows from Proposition 3.5 and Theorem 3.3.

Theorem 3.8 implies that any unitary bordism class can be represented by a disjoint union of products of projective toric manifolds. Products of toric manifolds are toric, but disjoint unions are not, as toric manifolds are connected. In bordism theory, a disjoint union may be replaced by a connected sum, representing the same bordism class. However, connected sum is not an algebraic operation, and a connected sum of two algebraic varieties is rarely algebraic. This can be remedied by appealing to quasitoric manifolds, as explained next. Recall that an omnioriented quasitoric manifold has an intrinsic stably complex structure, arising from the isomorphism of Theorem 2.7. One can form the equivariant connected sum of quasitoric manifolds, as explained in Davis and Januszkiewicz [8], but the resulting invariant stably complex structure does not represent the cobordism sum of the two original manifolds. A more intricate connected sum construction is needed, as outlined below. The details can be found in [3] or [2, Section 9.1].

Construction 3.9 The construction applies to two omnioriented $2 n$-dimensional quasitoric manifolds $M$ and $M^{\prime}$ over $n$-polytopes $P$ and $P^{\prime}$ respectively. The connected sum will be taken at the fixed points of $M$ and $M^{\prime}$ corresponding to vertices $v \in P$ and $v^{\prime} \in P^{\prime}$. We need to assume that $v$ is the intersection of the first $n$ facets of $P$, ie $v=F_{1} \cap \cdots \cap F_{n}$, and the corresponding characteristic matrix (2-5) of $M$ is in the refined form, ie

$$
\Lambda=\left(I \mid \Lambda_{\star}\right)=\left(\begin{array}{cccccc}
1 & 0 & 0 & \lambda_{1, n+1} & \cdots & \lambda_{1, m} \\
0 & \ddots & 0 & \vdots & \ddots & \vdots \\
0 & 0 & 1 & \lambda_{n, n+1} & \cdots & \lambda_{n, m}
\end{array}\right),
$$

where $I$ is the unit matrix and $\Lambda_{\star}$ is an $n \times(m-n)$-matrix. The same assumptions are made for $M^{\prime}, P^{\prime}, v^{\prime}$ and $\Lambda^{\prime}$.

The next step depends on the signs of the fixed points, $\sigma(v)$ and $\sigma\left(v^{\prime}\right)$. The sign of $v$ is determined by the omniorientation data; it is +1 when the orientation of $\mathcal{T}_{v} M$ induced from the global orientation of $M$ coincides with the orientation arising from $\left.\rho_{1} \oplus \cdots \oplus \rho_{n}\right|_{v}$, and is -1 otherwise. 
If $\sigma(v)=-\sigma\left(v^{\prime}\right)$, then we take the connected sum $M \# M^{\prime}$ at $v$ and $v^{\prime}$. It is a quasitoric manifold over $P \# P^{\prime}$ with the characteristic matrix $\left(\Lambda_{\star}|I| \Lambda_{\star}^{\prime}\right)$.

If $\sigma(v)=\sigma\left(v^{\prime}\right)$, then we need an additional connected summand. Consider the quasitoric manifold $S=S^{2} \times \cdots \times S^{2}$ over the $n$-cube $I^{n}$, where each $S^{2}$ is the quasitoric manifold over the segment $I$ with the characteristic matrix (11). It represents zero in $\Omega^{\mathrm{U}}$, and may be thought of as $\mathbb{C} P^{1}$ with the stably complex structure given by the isomorphism $\mathcal{T} \mathbb{C} P^{1} \oplus \underline{\mathbb{R}}^{2} \cong \bar{\eta} \oplus \eta$. The characteristic matrix of $S$ is therefore $(I \mid I)$. Now consider the connected sum $M \# S \# M^{\prime}$. It is a quasitoric manifold over $P \# I^{n} \# P^{\prime}$ with the characteristic matrix $\left(\Lambda_{\star}|I| I \mid \Lambda_{\star}^{\prime}\right)$.

In either case, the resulting omnioriented quasitoric manifold $M \# M^{\prime}$ or $M \# S \# M^{\prime}$ with the canonical stably complex structure represents the sum of bordism classes $[M]+\left[M^{\prime}\right] \in \Omega_{2 n}^{\mathrm{U}}$.

The conclusion, which can be derived from the above construction and any of the toric generating sets $\left\{B\left(n_{1}, n_{2}\right)\right\}$ or $\left\{L\left(n_{1}, n_{2}\right)\right\}$ for $\Omega^{\mathrm{U}}$, is as follows:

Theorem 3.10 [3] In dimensions $>2$, every unitary bordism class contains a quasitoric manifold, necessarily connected, whose stably complex structure is induced by an omniorientation, and is therefore compatible with the torus action.

\section{Special unitary bordism}

\section{Basics}

A special unitary structure (an SU-structure for short) on a manifold $M$ is a stably complex structure $c_{\mathcal{T}}$ with a choice of an SU-structure on the complex bundle $\xi$; see (3-1). A stably complex manifold $\left(M, c_{\mathcal{T}}\right)$ admits an SU-structure if and only if $c_{1}(\xi)=0$. Bordism classes of SU-manifolds form the special unitary bordism $\operatorname{ring} \Omega^{\mathrm{SU}}$.

The ring structure of $\Omega^{\mathrm{SU}}$ is more subtle than that of $\Omega^{\mathrm{U}}$. Novikov [13] described $\Omega^{\mathrm{SU}} \otimes \mathbb{Z}\left[\frac{1}{2}\right]$ (it is a polynomial ring). The 2 -torsion was described by Conner and Floyd [7]. For the description of the ring structure in $\Omega^{\mathrm{SU}}$ (which is not a polynomial ring, even modulo torsion), see [16]. We shall need the following facts:

Theorem $4.1 \quad$ (a) The kernel of the forgetful map $\Omega^{\mathrm{SU}} \rightarrow \Omega^{\mathrm{U}}$ consists of torsion elements.

(b) Every torsion element in $\Omega^{\mathrm{SU}}$ has order 2 .

(c) $\Omega^{\mathrm{SU}} \otimes \mathbb{Z}\left[\frac{1}{2}\right]$ is a polynomial algebra on generators in every even degree $>2$ :

$$
\Omega^{\mathrm{SU}} \otimes \mathbb{Z}\left[\frac{1}{2}\right] \cong \mathbb{Z}\left[\frac{1}{2}\right]\left[y_{i}: i>1\right], \quad \operatorname{deg} y_{i}=2 i .
$$


For the further analysis of the ring $\Omega^{\mathrm{SU}}$ we need to consider an auxiliary ring $\mathcal{W}$, apparently named after C T C Wall. We describe it following [7] and [16].

Let $\partial: \Omega_{2 n}^{\mathrm{U}} \rightarrow \Omega_{2 n-2}^{\mathrm{U}}$ be the homomorphism sending a bordism class $\left[M^{2 n}\right]$ to the bordism class $\left[V^{2 n-2}\right]$ of a submanifold $V^{2 n-2} \subset M$ dual to $c_{1}(M)$. There is a line bundle $\gamma$ over $M$ corresponding to $c_{1}(M)$, and the restriction of $\gamma$ to $V$ is the normal bundle $v(V \subset M)$. The stably complex structure on $V$ is defined via the isomorphism $\left.\mathcal{T} M\right|_{V} \cong \mathcal{T} V \oplus v(V \subset M)$. Then $V$ is an SU-manifold, so $\partial^{2}=0$. The homomorphism $\partial$ is not a derivation of $\Omega^{\mathrm{U}}$ though; it satisfies the identity

$$
\partial(a \cdot b)=a \cdot \partial b+\partial a \cdot b-\left[\mathbb{C} P^{1}\right] \cdot \partial a \cdot \partial b .
$$

Let $\mathcal{W}_{2 n}$ be the subgroup of $\Omega_{2 n}^{\mathrm{U}}$ consisting of bordism classes $\left[M^{2 n}\right]$ such that every Chern number of $M^{2 n}$ of which $c_{1}^{2}$ is a factor vanishes. The forgetful homomorphism decomposes as $\Omega_{2 n}^{\mathrm{SU}} \rightarrow \mathcal{W}_{2 n} \rightarrow \Omega_{2 n}^{\mathrm{U}}$, and the restriction of the boundary homomorphism д: $\mathcal{W}_{2 n} \rightarrow \mathcal{W}_{2 n-2}$ is defined.

The direct sum $\mathcal{W}=\bigoplus_{i \geqslant 0} \mathcal{W}_{2 i}$ is not a subring of $\Omega^{\mathrm{U}}$ : one has $\left[\mathbb{C} P^{1}\right] \in \mathcal{W}_{2}$, but $c_{1}^{2}\left[\mathbb{C} P^{1} \times \mathbb{C} P^{1}\right]=8 \neq 0$, so $\left[\mathbb{C} P^{1}\right] \times\left[\mathbb{C} P^{1}\right] \notin \mathcal{W}_{4}$. However, $\mathcal{W}$ becomes a commutative ring with unit with respect to the twisted product

$$
a * b=a \cdot b+2\left[V^{4}\right] \cdot \partial a \cdot \partial b,
$$

where - denotes the product in $\Omega^{\mathrm{U}}$ and where $V^{4}$ is a stably complex manifold with $c_{1}^{2}\left[V^{4}\right]=-1$. One may take $V^{4}=\mathbb{C} P^{1} \times \mathbb{C} P^{1}-\mathbb{C} P^{2}$ with the standard complex structure, or $V^{4}=\overline{\mathbb{C P}}^{2}$ with the stably complex structure defined by the isomorphism $\mathcal{T} \mathbb{C} P^{2} \oplus \underline{\mathbb{R}}^{2} \cong \bar{\eta} \oplus \bar{\eta} \oplus \eta$.

We shall use the notation

$$
m_{i}= \begin{cases}1 & \text { if } i+1 \neq p^{s} \text { for any prime } p, \\ p & \text { if } i+1=p^{s} \text { for some prime } p \text { and integer } s>0,\end{cases}
$$

so that $\left[M^{2 i}\right] \in \Omega_{2 i}^{\mathrm{U}}$ represents a polynomial generator whenever $s_{i}\left[M^{2 i}\right]= \pm m_{i}$.

Theorem 4.2 $\mathcal{W}$ is a polynomial ring on generators in every even degree except 4 :

$$
\mathcal{W} \cong \mathbb{Z}\left[x_{1}, x_{i}: i>2\right], \quad x_{1}=\left[\mathbb{C} P^{1}\right], \quad \operatorname{deg} x_{i}=2 i,
$$

with $s_{i}\left[x_{i}\right]=m_{i} m_{i-1}$, and the boundary operator $\partial: \mathcal{W} \rightarrow \mathcal{W}, \partial^{2}=0$, given by

$$
\partial x_{1}=2, \quad \partial x_{2 i}=x_{2 i-1},
$$

satisfies the identity

$$
\partial(a * b)=a * \partial b+\partial a * b-x_{1} * \partial a * \partial b
$$


The forgetful map $\alpha: \Omega^{\mathrm{SU}} \rightarrow \mathcal{W}$ is a ring homomorphism; this follows from (4-1) because $\partial \alpha(x)=0$ for any $x \in \Omega^{\mathrm{SU}}$.

The fundamental result relating $\Omega^{\mathrm{SU}}$ and $\mathcal{W}$ is as follows:

Theorem 4.3 There is an exact sequence of groups

$$
0 \longrightarrow \Omega_{2 n-1}^{\mathrm{SU}} \stackrel{\theta}{\longrightarrow} \Omega_{2 n}^{\mathrm{SU}} \stackrel{\alpha}{\longrightarrow} \mathcal{W}_{2 n} \stackrel{\beta}{\longrightarrow} \Omega_{2 n-2}^{\mathrm{SU}} \stackrel{\theta}{\longrightarrow} \Omega_{2 n-1}^{\mathrm{SU}} \longrightarrow 0,
$$

where $\theta$ is the multiplication by the generator $\theta \in \Omega_{1}^{\mathrm{SU}} \cong \mathbb{Z}_{2}$, $\alpha$ is the forgetful homomorphism, and $\alpha \beta=-\partial$.

Analysing the exact sequence above, one obtains the following information about the free and torsion parts of $\Omega^{\mathrm{SU}}$ :

Theorem 4.4 (a) $\operatorname{Torsion}\left(\Omega_{n}^{\mathrm{SU}}\right)=0$ unless $n=8 k+1$ or $8 k+2$, in which case $\operatorname{Torsion}\left(\Omega_{n}^{\mathrm{SU}}\right)$ is a $\mathbb{Z}_{2}$-vector space of rank equal to the number of partitions of $k$.

(b) $\Omega_{2 i}^{\mathrm{SU}} /$ Torsion is isomorphic to $\operatorname{Ker}(\partial: \mathcal{W} \rightarrow \mathcal{W})$ if $2 i \not \equiv 4 \bmod 8$ and is isomorphic to $\operatorname{Im}(\partial: \mathcal{W} \rightarrow \mathcal{W})$ if $2 i \equiv 4 \bmod 8$.

(c) There exist SU-bordism classes $w_{4 k} \in \Omega_{8 k}^{\mathrm{SU}}, k \geqslant 1$, such that $\operatorname{Im} \alpha / \operatorname{Im} \partial \cong$ $\mathbb{Z}_{2}\left[w_{4 k}\right]$. Every torsion element of $\Omega^{\mathrm{SU}}$ is uniquely expressible in the form $P \cdot \theta$ or $P \cdot \theta^{2}$ where $P$ is a polynomial in $w_{4 k}$ with coefficients 0 or 1 .

Note that we have

$$
\mathcal{W} \otimes \mathbb{Z}\left[\frac{1}{2}\right] \cong \mathbb{Z}\left[\frac{1}{2}\right]\left[x_{1}, x_{2 k-1}, 2 x_{2 k}-x_{1} x_{2 k-1}: k>1\right]
$$

where $x_{1}^{2}=x_{1} * x_{1}$ is a $\partial$-cycle, and each $x_{2 k-1}, 2 x_{2 k}-x_{1} x_{2 k-1}$ is a $\partial$-cycle.

Theorem 4.5 There exist elements $y_{i} \in \Omega_{2 i}^{\mathrm{SU}}, i>1$, such that $s_{i}\left(y_{i}\right)=m_{i} m_{i-1}$ if $i$ is odd, $s_{2}\left(y_{2}\right)=-48$, and $s_{i}\left(y_{i}\right)=2 m_{i} m_{i-1}$ if $i$ is even and $i>2$. These elements are mapped as follows under the forgetful homomorphism $\alpha: \Omega^{\mathrm{SU}} \rightarrow \mathcal{W}$ :

$$
y_{2} \mapsto 2 x_{1}^{2}, \quad y_{2 k-1} \mapsto x_{2 k-1}, \quad y_{2 k} \mapsto 2 x_{2 k}-x_{1} x_{2 k-1}, \quad k>1,
$$

where the $x_{i}$ are polynomial generators of $\mathcal{W}$. In particular, $\Omega^{\mathrm{SU}} \otimes \mathbb{Z}\left[\frac{1}{2}\right]$ embeds into (4-2) as the polynomial subring generated by $x_{1}^{2}, x_{2 k-1}$ and $2 x_{2 k}-x_{1} x_{2 k-1}$. 


\section{Quasitoric SU-manifolds}

Omnioriented quasitoric manifolds whose stably complex structures are SU can be detected using the following simple criterion:

Proposition 4.6 [4] An omnioriented quasitoric manifold $M$ has $c_{1}(M)=0$ if and only if there exists a linear function $\varphi: \mathbb{Z}^{n} \rightarrow \mathbb{Z}$ such that $\varphi\left(\lambda_{i}\right)=1$ for $i=1, \ldots, m$. Here the $\lambda_{i}$ are the columns of matrix (2-5).

In particular, if some $n$ vectors of $\lambda_{1}, \ldots, \lambda_{m}$ form the standard basis $\boldsymbol{e}_{1}, \ldots, \boldsymbol{e}_{n}$, then $M$ is SU if and only if the column sums of $\Lambda$ are all equal to 1 .

Proof By Theorem 2.7, $c_{1}(M)=v_{1}+\cdots+v_{m}$. By Theorem 2.6, $v_{1}+\cdots+v_{m}$ is zero in $H^{2}(M)$ if and only if

$$
v_{1}+\cdots+v_{m}=\sum_{i} \varphi\left(\lambda_{i}\right) v_{i}
$$

for some linear function $\varphi: \mathbb{Z}^{n} \rightarrow \mathbb{Z}$, whence the result follows.

Corollary 4.7 A toric manifold $V$ cannot be SU.

Proof If $\varphi\left(\lambda_{i}\right)=1$ for all $i$, then the vectors $\lambda_{i}$ lie in the positive halfspace of $\varphi$, so they cannot span a complete fan.

A more subtle result also rules out low-dimensional quasitoric manifolds:

Theorem 4.8 [4, Theorem 6.13] A quasitoric SU-manifold $M^{2 n}$ represents 0 in $\Omega_{2 n}^{\mathrm{U}}$ whenever $n<5$.

The reason for this is that the Krichever genus $\varphi_{\mathrm{K}}: \Omega^{\mathrm{U}} \rightarrow R_{\mathrm{K}}$ vanishes on quasitoric SU-manifolds, but $\varphi_{\mathrm{K}}$ is an isomorphism in dimensions $<10$.

Examples of quasitoric SU-manifolds representing nonzero bordism classes in $\Omega_{2 n}^{\mathrm{U}}$ for all $n \geqslant 5$, except $n=6$, were constructed in [11]. We modify this construction to present two particular families of quasitoric SU-manifolds representing nonzero bordism classes in $\Omega_{2 n}^{\mathrm{U}}$ for all $n \geqslant 5$, including $n=6$.

Construction 4.9 Assume that $n_{1}=2 k_{1}$ is positive even and $n_{2}=2 k_{2}+1$ is positive odd, and consider the manifold $L\left(n_{1}, n_{2}\right)$ from Construction 3.6. We change the stably complex structure (3-5) to the following:

$\mathcal{T} L\left(n_{1}, n_{2}\right) \oplus \underline{\mathbb{R}}^{4}$

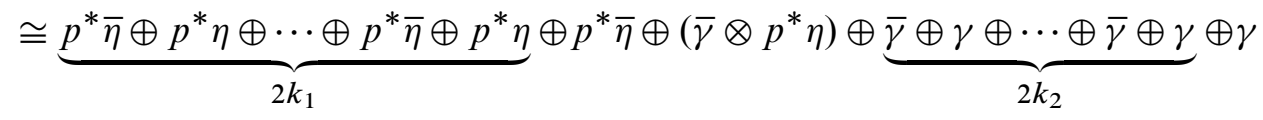


and denote the resulting stably complex manifold by $\widetilde{L}\left(n_{1}, n_{2}\right)$. Its cohomology ring is given by the same formula (3-4), but

$$
c\left(\tilde{L}\left(n_{1}, n_{2}\right)\right)=\left(1-u^{2}\right)^{k_{1}}(1+u)(1+v-u)\left(1-v^{2}\right)^{k_{2}}(1-v),
$$

so $\widetilde{L}\left(n_{1}, n_{2}\right)$ is an SU-manifold of dimension

$$
2\left(n_{1}+n_{2}\right)=4\left(k_{1}+k_{2}\right)+2 .
$$

Viewing $L\left(n_{1}, n_{2}\right)$ as a quasitoric manifold with the omniorientation coming from the complex structure, we see that changing a line bundle $\rho_{i}$ in (2-6) to its conjugate results in changing $\lambda_{i}$ to $-\lambda_{i}$ in (2-5). By applying this operation to the corresponding columns of (3-3) and then multiplying from the left by an appropriate matrix from GL $(n, \mathbb{Z})$, we obtain that $\tilde{L}\left(n_{1}, n_{2}\right)$ is the omnioriented quasitoric manifold over $\Delta^{n_{1}} \times \Delta^{n_{2}}$ corresponding to the matrix

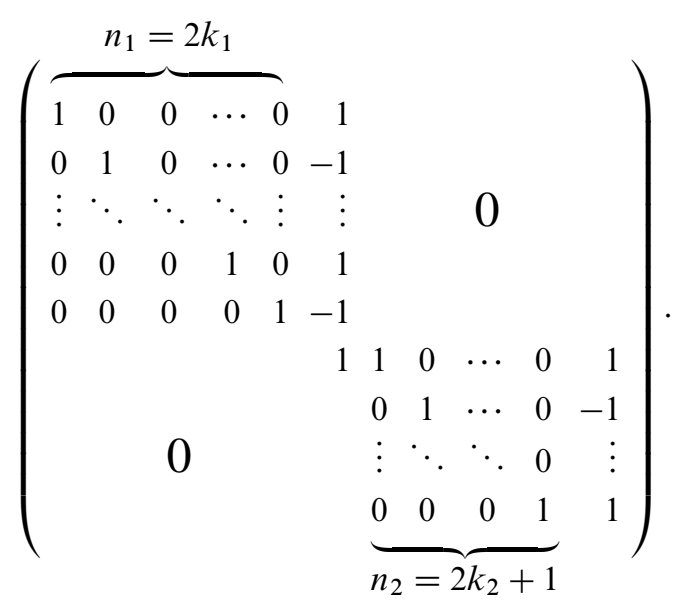

The column sums of this matrix are 1 by inspection.

Construction 4.10 The previous construction can be iterated by considering projectivisations of sums of line bundles over $L\left(n_{1}, n_{2}\right)$. We shall need just one particular family of this sort.

Given positive even $n_{1}=2 k_{1}$ and odd $n_{2}=2 k_{2}+1$, consider the omnioriented quasitoric manifold $\tilde{N}\left(n_{1}, n_{2}\right)$ over $\Delta^{1} \times \Delta^{n_{1}} \times \Delta^{n_{2}}$ with the characteristic matrix shown in Figure 1. The column sums are 1 by inspection, so $\tilde{N}\left(n_{1}, n_{2}\right)$ is a quasitoric SU-manifold of dimension $2\left(1+n_{1}+n_{2}\right)=4\left(k_{1}+k_{2}\right)+4$.

It can be seen that $\tilde{N}\left(n_{1}, n_{2}\right)$ is a projectivisation of a sum of $n_{2}+1$ line bundles over $\mathbb{C} P^{1} \times \mathbb{C} P^{n_{1}}$ with an amended stably complex structure. 


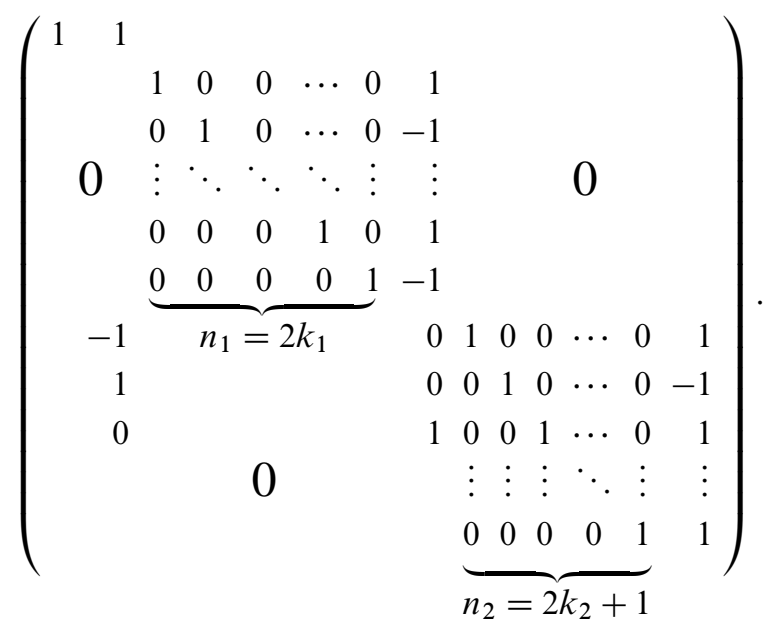

Figure 1: Characteristic matrix for the omnioriented quasitoric manifold $\tilde{N}\left(n_{1}, n_{2}\right)$ over $\Delta^{1} \times \Delta^{n_{1}} \times \Delta^{n_{2}}$

The cohomology ring given by Theorem 2.6 is

$$
H^{*}\left(\tilde{N}\left(n_{1}, n_{2}\right)\right) \cong \mathbb{Z}[u, v, w] /\left(u^{2}, v^{n_{1}+1},(w-u)^{2}(v+w) w^{n_{2}-2}\right)
$$

with $u v^{n_{1}} w^{n_{2}}\left\langle\tilde{N}\left(n_{1}, n_{2}\right)\right\rangle=1$. The total Chern class is

(4-5) $c\left(\tilde{N}\left(n_{1}, n_{2}\right)\right)=\left(1-v^{2}\right)^{k_{1}}(1+v)\left(1-(w-u)^{2}\right)(1-v-w)\left(1-w^{2}\right)^{k_{2}-1}(1+w)$.

\section{Quasitoric representatives for polynomial generators of $\Omega^{\mathrm{SU}} \otimes \mathbb{Z}\left[\frac{1}{2}\right]$}

Our goal is to show that elements $y_{i} \in \Omega_{2 i}^{\mathrm{SU}}$ described in Theorem 4.5 can be represented by quasitoric SU-manifolds when $i \geqslant 5$. This will be done by calculating the characteristic numbers of $\widetilde{L}\left(n_{1}, n_{2}\right)$ and $\widetilde{N}\left(n_{1}, n_{2}\right)$ and then checking several divisibility conditions for binomial coefficients. We shall need the following generalisation of Lucas' theorem:

Theorem 4.11 [9, Theorem 1] Suppose that prime power $p^{q}$ and positive integers $m=n+r$ are given. Write

$$
n=n_{0}+n_{1} p+\cdots+n_{k-1} p^{k-1}+n_{k} p^{k}
$$

in base $p$, and let

$$
N_{j}=n_{j}+n_{j+1} p+\cdots+n_{j+q-1} p^{q-1}, \quad j \geqslant 0 .
$$


Also make the corresponding definitions for $m_{j}, M_{j}, r_{j}, R_{j}$. Let $e_{j}$ be the number of indices $i \geqslant j$ for which $n_{i}<m_{i}$. Then

$$
\frac{1}{p^{e_{0}}}\left(\begin{array}{c}
n \\
m
\end{array}\right) \equiv( \pm 1)^{e_{q-1}} \frac{N_{0} !_{p}}{M_{0} !_{p} R_{0} !_{p}} \cdot \frac{N_{1} !_{p}}{M_{1} !_{p} R_{1} !_{p}} \cdots \frac{N_{k} !_{p}}{M_{k} !_{p} R_{k} !_{p}} \quad \bmod p^{q},
$$

where \pm 1 is -1 except if $p=2$ and $q \geqslant 3$, and $n ! p$ denotes the product of those positive integers $\leqslant n$ that are not divisible by $p$.

Lemma 4.12 For $n_{1}=2 k_{1}>0$ and $n_{2}=2 k_{2}+1>0$, we have

$$
s_{n_{1}+n_{2}}\left[\tilde{L}\left(n_{1}, n_{2}\right)\right]=-\left(\begin{array}{c}
n_{1}+n_{2} \\
1
\end{array}\right)+\left(\begin{array}{c}
n_{1}+n_{2} \\
2
\end{array}\right)-\cdots-\left(\begin{array}{c}
n_{1}+n_{2} \\
n_{1}-1
\end{array}\right)+\left(\begin{array}{c}
n_{1}+n_{2} \\
n_{1}
\end{array}\right) .
$$

Proof Using (4-3) and (3-4) we calculate

$$
\begin{aligned}
s_{n_{1}+n_{2}}\left(\widetilde { L } \left(n_{1},\right.\right. & \left.\left.n_{2}\right)\right) \\
& =(v-u)^{n_{1}+n_{2}}+\left(k_{2}+1\right)(-1)^{n_{1}+n_{2}} v^{n_{1}+n_{2}}+k_{2} v^{n_{1}+n_{2}} \\
& =(v-u)^{n_{1}+n_{2}}-v^{n_{1}+n_{2}} \\
& =\left(-\left(\begin{array}{c}
n_{1}+n_{2} \\
1
\end{array}\right)+\left(\begin{array}{c}
n_{1}+n_{2} \\
2
\end{array}\right)-\cdots-\left(\begin{array}{c}
n_{1}+n_{2} \\
n_{1}-1
\end{array}\right)+\left(\begin{array}{c}
n_{1}+n_{2} \\
n_{1}
\end{array}\right)\right) u^{n_{1}} v^{n_{2}},
\end{aligned}
$$

and the result follows by evaluating at $\left\langle\tilde{L}\left(n_{1}, n_{2}\right)\right\rangle$.

Note that $s_{3}(\tilde{L}(2,1))=0$ in accordance with Theorem 4.8. On the other hand, $s_{2+n_{2}}\left(\tilde{L}\left(2, n_{2}\right)\right) \neq 0$ for $n_{2}>1$, providing an example of a non-bounding quasitoric SU-manifold in each dimension $4 k+2$ with $k>1$.

Lemma 4.13 For $k>1$, there is a linear combination $y_{2 k+1}$ of SU-bordism classes $\left[\tilde{L}\left(n_{1}, n_{2}\right)\right]$ with $n_{1}+n_{2}=2 k+1$ such that $s_{2 k+1}\left(y_{2 k+1}\right)=m_{2 k+1} m_{2 k}$.

Proof By the previous lemma,

$$
s_{n_{1}+n_{2}}\left[\widetilde{L}\left(n_{1}, n_{2}\right)-\tilde{L}\left(n_{1}-2, n_{2}+2\right)\right]=\left(\begin{array}{c}
n_{1}+n_{2} \\
n_{1}
\end{array}\right)-\left(\begin{array}{c}
n_{1}+n_{2} \\
n_{1}-1
\end{array}\right) .
$$

The result follows from the next lemma.

Lemma 4.14 For any integer $k>1$, we have

$$
\operatorname{gcd}\left\{\left(\begin{array}{c}
2 k+1 \\
2 i
\end{array}\right)-\left(\begin{array}{c}
2 k+1 \\
2 i-1
\end{array}\right), 0<i \leqslant k\right\}=m_{2 k+1} m_{2 k} .
$$

Remark This result has been obtained independently in a recent work of Buchstaber and Ustinov on the coefficient rings of universal formal group laws [6, Section 9]. 
Proof of Lemma 4.14 We need to establish the following two facts:

(a) The largest power of 2 which divides each number $\left(\begin{array}{c}2 k+1 \\ 2 i\end{array}\right)-\left(\begin{array}{c}2 k+1 \\ 2 i-1\end{array}\right)$ with $0<i \leqslant k$ is 2 if $2 k+2=2^{s}$ and is 1 otherwise.

(b) The largest power of odd prime $p$ which divides each number $\left(\begin{array}{c}2 k+1 \\ 2 i\end{array}\right)-\left(\begin{array}{c}2 k+1 \\ 2 i-1\end{array}\right)$ with $0<i \leqslant k$ is $p$ if $2 k+1=p^{s}$ and is 1 otherwise.

We prove (a) first.

Case $1\left(2 k+2=2^{s}\right)$ Then $s>2$, as $k>1$. For $0<i \leqslant k$, we have

$$
\left(\begin{array}{c}
2 k+1 \\
2 i
\end{array}\right)-\left(\begin{array}{c}
2 k+1 \\
2 i-1
\end{array}\right) \equiv\left(\begin{array}{c}
2^{s}-1 \\
2 i
\end{array}\right)+\left(\begin{array}{c}
2^{s}-1 \\
2 i-1
\end{array}\right)=\left(\begin{array}{l}
2^{s} \\
2 i
\end{array}\right) \equiv 0 \bmod 2
$$

by Proposition 3.5. On the other hand,

$$
\left(\begin{array}{c}
2^{s}-1 \\
2
\end{array}\right)-\left(\begin{array}{c}
2^{s}-1 \\
1
\end{array}\right)=\left(2^{s}-1\right)\left(2^{s-1}-1-1\right)=2\left(2^{s}-1\right)\left(2^{s-2}-1\right) \not \equiv 0 \quad \bmod 4
$$

Case $2\left(2 k+2 \neq 2^{s}\right)$ Write the base 2 expansion

$$
2 k+2=n_{1} 2+\cdots+n_{l-1} 2^{l-1}+2^{l}
$$

with $n_{i}=1$ or 0 . Set $2 i=n_{1} 2+\cdots+n_{l-1} 2^{l-1}$. Then we have $2 i \neq 0$, as otherwise $2 k+2=2^{l}$. Then $\left(\begin{array}{c}2 k+1 \\ 2 i\end{array}\right)-\left(\begin{array}{c}2 k+1 \\ 2 i-1\end{array}\right) \equiv\left(\begin{array}{c}2 k+2 \\ 2 i\end{array}\right) \equiv 1 \bmod 2$ by Theorem 3.4.

Now we prove (b).

Case $1\left(2 k+1=p^{s}\right)$ Then $\left(\begin{array}{c}2 k+1 \\ 2 i\end{array}\right)-\left(\begin{array}{c}2 k+1 \\ 2 i-1\end{array}\right) \equiv 0 \bmod p$ for $0<i \leqslant k$ by Proposition 3.5. On the other hand, setting $2 i=p^{s-1}+1$, we get

$$
\begin{aligned}
\left(\begin{array}{c}
2 k+1 \\
2 i
\end{array}\right)-\left(\begin{array}{c}
2 k+1 \\
2 i-1
\end{array}\right) & =\frac{2 k-4 i+2}{2 i}\left(\begin{array}{c}
2 k+1 \\
2 i-1
\end{array}\right) \\
& =\frac{p^{s}-2 p^{s-1}-1}{p^{s-1}+1}\left(\begin{array}{c}
p^{s} \\
p^{s-1}
\end{array}\right) \not \equiv 0 \bmod p^{2} .
\end{aligned}
$$

This follows from the fact that $p^{s}-2 p^{s-1}-1>0$ as $k>1$, and $\left(\begin{array}{c}p^{s} \\ p^{s-1}\end{array}\right)$ is not divisible by $p^{2}$ by Kummer's theorem.

Case $2\left(2 k+1 \neq p^{s}\right)$ Write the base $p$ expansion

$$
2 k+1=n_{0}+n_{1} p+\cdots+n_{l-1} p^{l-1}+n_{l} p^{l}
$$

with $0 \leqslant n_{i} \leqslant p-1$ (for $i=0, \ldots, l$ ) and $n_{l}>0$.

Assume that $n_{0}>1$. Then we set

$$
2 i=n_{0}+n_{1} p+\cdots+n_{l-1} p^{l-1}+\left(n_{l}-1\right) p^{l} .
$$


We have $\left(\begin{array}{c}2 k+1 \\ 2 i\end{array}\right) \equiv n_{l} \bmod p$ by Theorem 3.4. Also,

$$
2 i-1=\left(n_{0}-1\right)+n_{1} p+\cdots+n_{l-1} p^{l-1}+\left(n_{l}-1\right) p^{l}>0
$$

and $\left(\begin{array}{c}2 k+1 \\ 2 i-1\end{array}\right) \equiv n_{l} n_{0} \bmod p$. Therefore, $\left(\begin{array}{c}2 k+1 \\ 2 i\end{array}\right)-\left(\begin{array}{c}2 k+1 \\ 2 i-1\end{array}\right) \equiv n_{l}\left(1-n_{0}\right) \not \equiv 0 \bmod p$.

Assume that $n_{0}=1$. Then we set $2 i=2 k$. We have

$\left(\begin{array}{c}2 k+1 \\ 2 k\end{array}\right)=2 k+1 \equiv 1 \quad \bmod p \quad$ and $\quad\left(\begin{array}{c}2 k+1 \\ 2 k-1\end{array}\right)=k(2 k+1) \equiv 0 \quad \bmod p$,

so that

$$
\left(\begin{array}{c}
2 k+1 \\
2 k
\end{array}\right)-\left(\begin{array}{c}
2 k+1 \\
2 k-1
\end{array}\right) \not \equiv 0 \bmod p
$$

Finally, assume that $n_{0}=0$. Then we set

$$
\begin{aligned}
2 i & =n_{0}+n_{1} p+\cdots+n_{l-1} p^{l-1}+\left(n_{l}-1\right) p^{l} \\
& =n_{q} p^{q}+\cdots+n_{l-1} p^{l-1}+\left(n_{l}-1\right) p^{l},
\end{aligned}
$$

where $q>0$ and $n_{q}>0$. We have $2 i>0$, as otherwise $2 k+1=p^{l}$. Then $\left(\begin{array}{c}2 k+1 \\ 2 i\end{array}\right) \equiv n_{l} \bmod p$. Also,

$2 i-1=(p-1)+(p-1) p+\cdots+(p-1) p^{q-1}+\left(n_{q}-1\right) p^{q}+\cdots+n_{l-1} p^{l-1}+\left(n_{l}-1\right) p^{l}$, and $\left(\begin{array}{c}2 k+1 \\ 2 i-1\end{array}\right) \equiv 0 \bmod p$ by Theorem 3.4. Therefore, $\left(\begin{array}{c}2 k+1 \\ 2 i\end{array}\right)-\left(\begin{array}{c}2 k+1 \\ 2 i-1\end{array}\right) \not \equiv 0 \bmod p$.

Now we turn our attention to the manifolds $\tilde{N}\left(n_{1}, n_{2}\right)$ from Construction 4.10.

Lemma 4.15 For $n_{1}=2 k_{1}>0$ and $n_{2}=2 k_{2}+1>0$, set $n=n_{1}+n_{2}+1$, so that $\operatorname{dim} \tilde{N}\left(n_{1}, n_{2}\right)=2 n=4\left(k_{1}+k_{2}+1\right)$. Then

$$
s_{n}\left[\tilde{N}\left(n_{1}, n_{2}\right)\right]=2\left(-\left(\begin{array}{c}
n \\
1
\end{array}\right)+\left(\begin{array}{c}
n \\
2
\end{array}\right)-\cdots-\left(\begin{array}{c}
n \\
n_{1}-1
\end{array}\right)+\left(\begin{array}{c}
n \\
n_{1}
\end{array}\right)-n_{1}\right) .
$$

Proof Using (4-5) and (4-4) we calculate

(4-6) $\quad s_{n}\left(\tilde{N}\left(n_{1}, n_{2}\right)\right)$

$$
\begin{aligned}
& =2(w-u)^{n}+(v+w)^{n}+\left(2 k_{2}-1\right) w^{n} \\
& =2 w^{n}-2 n u w^{n-1}+w^{n}+\left(\begin{array}{c}
n \\
1
\end{array}\right) v w^{n-1}+\cdots \\
& \quad+\left(\begin{array}{c}
n \\
2 k_{1}
\end{array}\right) v^{2 k_{1}} w^{2 k_{2}+2}+\left(2 k_{2}-1\right) w^{n} \\
& \quad+2 n u w^{n-1}+\left(n-n_{1}\right) w^{n}+\left(\begin{array}{c}
n \\
1
\end{array}\right) v w^{n-1}+\cdots+\left(\begin{array}{c}
n \\
n_{1}
\end{array}\right) v^{n_{1}} w^{n-n_{1}} .
\end{aligned}
$$


Now we have to express each monomial above via $u v^{n_{1}} w^{n_{2}}$ using the identities in (4-4), namely

$$
u^{2}=0, \quad v^{n_{1}+1}=0, \quad w^{n_{2}+1}=2 u w^{n_{2}}-v w^{n_{2}}+2 u v w^{n_{2}-1} .
$$

We have

$$
\begin{aligned}
u w^{n-1} & =u w^{n_{1}-1} w^{n_{2}+1}=u w^{n_{1}-1}\left(2 u w^{n_{2}}-v w^{n_{2}}+2 u v w^{n_{2}-1}\right) \\
& =-u v w^{n-2}=\cdots=(-1)^{j} u v^{j} w^{n-j-1}=\cdots=u v^{n_{1}} w^{n_{2}} .
\end{aligned}
$$

Also, we show that

$$
v^{j} w^{n-j}=(-1)^{j} 2 u v^{n_{1}} w^{n_{2}}, \quad 0 \leqslant j \leqslant n_{1},
$$

by verifying the identity successively for $j=n_{1}, n_{1}-1, \ldots, 0$. Indeed,

$$
v^{n_{1}} w^{n-n_{1}}=v^{n_{1}} w^{n_{2}+1}=2 u v^{n_{1}} w^{n_{2}}
$$

by (4-7). Now, we have

$$
\begin{aligned}
v^{j-1} w^{n-j+1} & =v^{j-1} w^{n_{1}+1-j} w^{n_{2}+1}=v^{j-1} w^{n_{1}+1-j}\left(2 u w^{n_{2}}-v w^{n_{2}}+2 u v w^{n_{2}-1}\right) \\
& =2 u v^{j-1} w^{n-j}-v^{j} w^{n-j}+2 u v^{j} w^{n-1-j}=-v^{j} w^{n-j}
\end{aligned}
$$

where the last identity holds because of (4-8). The identity (4-9) is therefore verified completely. Plugging (4-8) and (4-9) into (4-6) we obtain

$s_{n}\left(\tilde{N}\left(n_{1}, n_{2}\right)\right)$

$$
=\left(-2 n+2\left(n-n_{1}\right)-2\left(\begin{array}{c}
n \\
1
\end{array}\right)+2\left(\begin{array}{l}
n \\
2
\end{array}\right)-\cdots-2\left(\begin{array}{c}
n \\
n_{1}-1
\end{array}\right)+2\left(\begin{array}{c}
n \\
n_{1}
\end{array}\right)\right) u v^{n_{1}} w^{n_{2}} .
$$

The result follows by evaluating at $\left\langle\tilde{N}\left(n_{1}, n_{2}\right)\right\rangle$.

Note that $s_{4}(\tilde{N}(2,1))=0$ in accordance with Theorem 4.8. On the other hand, $s_{n}\left(\tilde{N}\left(2, n_{2}\right)\right)=n^{2}-3 n-4>0$ for $n>4$, providing an example of a non-bounding quasitoric SU-manifold in each dimension $4 k$ with $k>2$. This includes a $12-$ dimensional quasitoric SU-manifold $\tilde{N}(2,3)$, which was missing in [11].

Lemma 4.16 For $k>2$, there is a linear combination $y_{2 k}$ of SU-bordism classes $\left[\tilde{N}\left(n_{1}, n_{2}\right)\right]$ with $n_{1}+n_{2}+1=2 k$ such that $s_{2 k}\left(y_{2 k}\right)=2 m_{2 k} m_{2 k-1}$.

Proof The result follows from Lemma 4.15 and Lemmata 4.17 and 4.18 below.

Lemma 4.17 For $k>2$, the largest power of 2 which divides each number

$$
a_{i}=-\left(\begin{array}{c}
2 k \\
1
\end{array}\right)+\left(\begin{array}{c}
2 k \\
2
\end{array}\right)-\cdots-\left(\begin{array}{c}
2 k \\
2 i-1
\end{array}\right)+\left(\begin{array}{c}
2 k \\
2 i
\end{array}\right)-2 i, \quad 0<i<k,
$$

is 2 if $2 k=2^{s}$ and is 1 otherwise. 
Proof First assume that $2 k=2^{s}$. Then $a_{i} \equiv 0 \bmod 2$ by Proposition 3.5. On the other hand, we have

$$
a_{1}=-2^{s}+2^{s-1}\left(2^{s}-1\right)-2 \not \equiv 0 \bmod 4,
$$

because $s>2$.

Now assume that $2 k \neq 2^{s}$. We have $a_{i}-a_{i-1} \equiv\left(\begin{array}{c}2 k \\ 2 i\end{array}\right) \bmod 2$, so it is enough to find $i$ such that $\left(\begin{array}{c}2 k \\ 2 i\end{array}\right) \neq 0 \bmod 2$. This was done in the proof of Lemma 4.14.

Lemma 4.18 For $k>2$, the largest power of odd prime $p$ which divides each

$$
a_{i}=-\left(\begin{array}{c}
2 k \\
1
\end{array}\right)+\left(\begin{array}{c}
2 k \\
2
\end{array}\right)+\cdots-\left(\begin{array}{c}
2 k \\
2 i-1
\end{array}\right)+\left(\begin{array}{c}
2 k \\
2 i
\end{array}\right)-2 i, \quad 0<i<k,
$$

is $p$ if $2 k+1=p^{s}$ and is 1 otherwise.

Proof Using the identity

$$
2+\sum_{j=1}^{2 k-1}(-1)^{j}\left(\begin{array}{c}
2 k \\
j
\end{array}\right)=0
$$

we obtain $a_{k-1}=0$ and

$$
a_{i}+a_{k-i-1}=\left(\begin{array}{c}
2 k \\
2 i+1
\end{array}\right)-2 k, \quad 0<i<k-1 .
$$

Case $1\left(2 k+1=p^{s}\right)$ We have

$$
\left(\begin{array}{c}
2 k \\
2 i+1
\end{array}\right)=\left(\begin{array}{c}
p^{s}-1 \\
2 i+1
\end{array}\right)=\left(\begin{array}{c}
p^{s}-1 \\
2 i-1
\end{array}\right) \frac{\left(p^{s}-2 i\right)\left(p^{s}-2 i-1\right)}{2 i(2 i+1)} \equiv-1 \quad \bmod p
$$

by induction starting from $i=0$. Therefore,

$$
a_{i}=a_{i-1}-\left(\begin{array}{c}
2 k \\
2 i-1
\end{array}\right)+\left(\begin{array}{c}
2 k \\
2 i
\end{array}\right)-2=a_{i-1}+\frac{p^{s}-4 i}{2 i}\left(\begin{array}{c}
p^{s}-1 \\
2 i-1
\end{array}\right)-2 \equiv 0 \quad \bmod p
$$

by induction starting from $a_{0}=0$. In view of (4-10), it suffices to find $i$, where $0<i<k-1$, such that

$$
\left(\begin{array}{c}
2 k \\
2 i+1
\end{array}\right)-2 k \not \equiv 0 \bmod p^{2}
$$

If $s=1$, then $p>5$ as $k>2$. We set $2 i+1=3$, so that

$$
\left(\begin{array}{c}
2 k \\
2 i+1
\end{array}\right)-2 k=\left(\begin{array}{c}
p-1 \\
3
\end{array}\right)-(p-1)=\frac{p(p-1)(p-5)}{6} \not \equiv 0 \bmod p^{2} .
$$


Now assume that $s>1$. We set $2 i+1=p^{s-1}$ and use Theorem 4.11 to calculate $\left(\begin{array}{c}2 k \\ 2 i+1\end{array}\right) \bmod p^{2}$. In the notation of Theorem 4.11, we have $q=2$,

$$
\begin{gathered}
n=p^{s}-1=n_{0}+n_{1} p+\cdots+n_{s-2} p^{s-2}+n_{s-1} p^{s-1} \\
=(p-1)+(p-1) p+\cdots+(p-1) p^{s-2}+(p-1) p^{s-1}, \\
N_{0}=\cdots=N_{s-2}=p^{2}-1, \quad N_{s-1}=p-1, \\
m=p^{s-1}=m_{0}+m_{1} p+\cdots+m_{s-2} p^{s-2}+m_{s-1} p^{s-1}, \\
M_{0}=\cdots=M_{s-3}=0, \quad M_{s-2}=p, \quad M_{s-1}=1, \\
r=p^{s-} p^{s-1}=r_{0}+r_{1} p+\cdots+r_{s-2} p^{s-2}+r_{s-1} p^{s-1} \\
=(p-1)+(p-1) p+\cdots+(p-1) p^{s-2}+(p-2) p^{s-1}, \\
R_{0}=\cdots=R_{s-3}=p^{2}-1, \quad R_{s-2}=p^{2}-p-1, \quad R_{s-1}=p-2,
\end{gathered}
$$

and $e_{0}=e_{1}=0$. Therefore, Theorem 4.11 gives

$$
\begin{aligned}
\left(\begin{array}{c}
p^{s}-1 \\
p^{s-1}
\end{array}\right) & \equiv \frac{\left(p^{2}-1\right) !_{p}}{p !_{p}\left(p^{2}-p-1\right) ! p} \cdot \frac{(p-1) !_{p}}{1 ! p(p-2) !_{p}}=\frac{\left(p^{2}-1\right) \cdots\left(p^{2}-p+1\right)}{(p-1) !} \cdot(p-1) \\
& \equiv p-1 \bmod p^{2},
\end{aligned}
$$

and we obtain

$$
\left(\begin{array}{c}
2 k \\
2 i+1
\end{array}\right)-2 k=\left(\begin{array}{c}
p^{s}-1 \\
p^{s-1}
\end{array}\right)-\left(p^{s}-1\right) \equiv p \quad \bmod p^{2} .
$$

Case $2\left(2 k+1 \neq p^{s}\right)$ In view of (4-10), it suffices to find $i$, where $0<i<k-1$, such that $\left(\begin{array}{c}2 k \\ 2 i+1\end{array}\right)-2 k \neq 0$ mod $p$. Write the base $p$ expansion

$$
2 k=n_{0}+n_{1} p+\cdots+n_{l-1} p^{l-1}+n_{l} p^{l}
$$

with $0 \leqslant n_{i} \leqslant p-1$ (for $i=0, \ldots, l$ ) and $n_{l}>0$. We have $2 k \equiv n_{0} \bmod p$.

Assume that $n_{0}=0$. Then we set

$$
2 i+1=n_{0}+n_{1} p+\cdots+n_{l-1} p^{l-1}+\left(n_{l}-1\right) p^{l} .
$$

We have $\left(\begin{array}{c}2 k \\ 2 i+1\end{array}\right)-2 k \equiv n_{l} \neq \equiv 0 \bmod p$.

Assume that $0<n_{0}<p-2$. If $2 k<p$, then $n_{0}=2 k>5$. We set $2 i+1=3$, so that

$$
\left(\begin{array}{c}
2 k \\
2 i+1
\end{array}\right)-2 k \equiv\left(\begin{array}{c}
n_{0} \\
3
\end{array}\right)-n_{0}=\frac{n_{0}\left(n_{0}-4\right)\left(n_{0}+1\right)}{6} \not \equiv 0 \bmod p .
$$


If $2 k>p$, then we set

$$
2 i+1= \begin{cases}n_{0}+1 & \text { if } n_{0} \text { is even } \\ n_{0}+2 & \text { if } n_{0} \text { is odd. }\end{cases}
$$

We have $2 i+1<2 k$ and $\left(\begin{array}{c}2 k \\ 2 i+1\end{array}\right)-2 k \equiv-n_{0} \neq \equiv 0 \bmod p$.

Assume that $n_{0}=p-2$. If $p=3$, then $n_{0}=1$. We set $2 i+1=5<2 k$, so that

$$
\left(\begin{array}{c}
2 k \\
2 i+1
\end{array}\right)-2 k \equiv\left(\begin{array}{c}
n_{0} \\
2
\end{array}\right)\left(\begin{array}{c}
n_{1} \\
1
\end{array}\right)-1=-1 \not \equiv 0 \bmod p .
$$

If $p>3$, then we set $2 i+1=3$, so that $\left(\begin{array}{c}2 k \\ 2 i+1\end{array}\right)-2 k \neq 0 \bmod p$ by (4-11).

Assume that $n_{0}=p-1$ and $n_{l}<p-1$. Then we set

$$
2 i+1=n_{0}+n_{1} p+\cdots+n_{l-1} p^{l-1}+\left(n_{l}-1\right) p^{l} .
$$

We have $\left(\begin{array}{c}2 k \\ 2 i+1\end{array}\right)-2 k \equiv n_{l}-n_{0} \not \equiv 0 \bmod p$.

Finally, assume that $n_{0}=p-1$ and $n_{l}=p-1$. As $2 k \neq p^{s}-1$, there exists $j$, where $0<j<l$, such that $n_{j}<p-1$. Then we set

$$
2 i+1=n_{0}+n_{1} p+\cdots+n_{j-1} p^{j-1}+\left(n_{j}+1\right) p^{j} .
$$

We have $2 i+1<2 k$ and $\left(\begin{array}{c}2 k \\ 2 i+1\end{array}\right)-2 k \equiv-n_{0} \not \equiv 0 \bmod p$.

We now can prove our main result:

Theorem 4.19 There exist quasitoric SU-manifolds $M^{2 i}, i \geqslant 5$, with $s_{i}\left(M^{2 i}\right)=$ $m_{i} m_{i-1}$ if $i$ is odd and $s_{i}\left(M^{2 i}\right)=2 m_{i} m_{i-1}$ if $i$ is even. These quasitoric manifolds represent polynomial generators of $\Omega^{\mathrm{SU}} \otimes \mathbb{Z}\left[\frac{1}{2}\right]$.

Proof It follows from Lemmata 4.13 and 4.16 that there exist linear combinations of SU-bordism classes represented by quasitoric SU-manifolds with the required properties. We observe that application of Construction 3.9 to two quasitoric SU-manifolds $M$ and $M^{\prime}$ produces a quasitoric SU-manifold representing their bordism sum. Also, the SU-bordism class $-[M]$ can be represented by the omnioriented quasi-toric SU-manifold obtained by reversing the global orientation of $M$. Therefore, we can replace the linear combinations obtained using Lemmata 4.13 and 4.16 by appropriate connected sums, which are quasitoric SU-manifolds. 


\section{Concluding remarks}

By analogy with Theorem 3.10, we may ask the following:

Question 4.20 Which SU-bordism classes of dimension $>8$ can be represented by quasitoric SU-manifolds?

Theorem 4.19 provides quasitoric representatives for the elements $y_{i} \in \Omega_{2 i}^{\mathrm{SU}}$ described in Theorem 4.5 for $i \geqslant 5$. The elements $y_{2}, y_{3}, y_{4}$ cannot be represented by quasitoric manifolds because of Theorem 4.8. Any polynomial in these elements cannot be represented by a quasitoric manifold for the same reason: the Krichever genus $\varphi_{\mathrm{K}}: \Omega^{\mathrm{U}} \rightarrow R_{\mathrm{K}}$ vanishes on quasitoric SU-manifolds, but $\varphi_{\mathrm{K}}$ is nonzero on any polynomial in $y_{2}, y_{3}$, and $y_{4}$. We thank Michael Wiemeler for this observation.

The element $x_{1}^{2} \in \mathcal{W}_{4}$ (see Theorem 4.2) is represented by $9 \mathbb{C} P^{1} \times \mathbb{C} P^{1}-8 \mathbb{C} P^{2}$, which is also the bordism class of a toric manifold over a 12-gon, with characteristic numbers $c_{1}^{2}=0$ and $c_{2}=12$ (so $s_{2}=-24$ ). The element $y_{2}=2 x_{1}^{2} \in \Omega_{4}^{\mathrm{SU}}$ is represented by a $K 3$ surface, but not by a toric manifold.

The 6-sphere $S^{6}$ has a $T^{2}$-invariant almost complex structure as the homogeneous space $G_{2} / \mathrm{SU}(3)$ of the exceptional Lie group $G_{2}$ (see [1]), and therefore represents an SU-bordism class in $\Omega_{6}^{\mathrm{SU}}$. Its characteristic numbers are $c_{1}^{3}=c_{1} c_{2}=0$ and $c_{3}=2$. Therefore, $s_{3}\left[S^{6}\right]=6=m_{3} m_{2}$, so $S^{6}$ represents $y_{3} \in \Omega_{6}^{\mathrm{SU}}$.

It would be interesting to find good geometric representatives for $y_{4} \in \Omega_{8}^{\mathrm{SU}}$, and also for the elements $w_{4 k} \in \Omega_{8 k}^{\mathrm{SU}}$ that control the 2-torsion in Theorem 4.4(c). The image of $w_{4 k}$ under the forgetful homomorphism $\alpha: \Omega_{8 k}^{\mathrm{SU}} \rightarrow \mathcal{W}_{8 k}$ is $x_{1}^{4 k}$, so it is decomposable in $\Omega^{\mathrm{U}}$ and has $s_{4 k}\left[w_{4 k}\right]=0$. The conditions on the characteristic numbers specifying $w_{4 k}$ are given in [7, (19.3)].

\section{References}

[1] A Borel, F Hirzebruch, Characteristic classes and homogeneous spaces, I, Amer. J. Math. 80 (1958) 458-538 MR0102800

[2] V M Buchstaber, T E Panov, Toric topology, Mathematical Surveys and Monographs 204, Amer. Math. Soc., Providence, RI (2015) MR3363157

[3] V M Buchstaber, T E Panov, N Ray, Spaces of polytopes and cobordism of quasitoric manifolds, Mosc. Math. J. 7 (2007) 219-242, 350 MR2337880

[4] V M Buchstaber, T Panov, N Ray, Toric genera, Int. Math. Res. Not. (2010) 32073262 MR2673724 
[5] V M Buchstaber, N Ray, Toric manifolds and complex cobordisms, Uspekhi Mat. Nauk 53 (1998) 139-140 MR1639388 In Russian; translated in Russ. Math. Surv. 53 (1998) 371-373

[6] V M Buchstaber, A V Ustinov, Coefficient rings of formal group laws, Mat. Sb. 206 (2015) 19-60 MR3438568 In Russian; translated in Sb. Math. 206 (2015) 1524-1563

[7] P E Conner, E E Floyd, Torsion in SU-bordism, Memoirs of the American Mathematical Society 60, Amer. Math. Soc, Providence, RI (1966) MR0189044

[8] M W Davis, T Januszkiewicz, Convex polytopes, Coxeter orbifolds and torus actions, Duke Math. J. 62 (1991) 417-451 MR1104531

[9] A Granville, Arithmetic properties of binomial coefficients, I: Binomial coefficients modulo prime powers, from "Organic mathematics", CMS Conf. Proc. 20, Amer. Math. Soc. (1997) 253-276 MR1483922

[10] Z Lü, Equivariant bordism of 2-torus manifolds and unitary toric manifolds - a survey, preprint (2014) arXiv:1401.3052

[11] Z Lü, W Wang, Examples of quasitoric manifolds as special unitary manifolds, preprint (2015) arXiv:1310.3933v3 To appear in Math. Res. Lett.

[12] M Masuda, D Y Suh, Classification problems of toric manifolds via topology, from "Toric topology" (M Harada, Y Karshon, M Masuda, T Panov, editors), Contemp. Math. 460, Amer. Math. Soc., Providence, RI (2008) 273-286 MR2428362

[13] S P Novikov, Homotopy properties of Thom complexes, Mat. Sb. 57 (99) (1962) 407442 MR0157381 In Russian; translation available at http://tinyurl.com/NovikovEng

[14] S D Oshanin, The signature of SU-manifolds, Mat. Zametki 13 (1973) 97-102 MR0339238 In Russian; translated in Math. Notes 13 (1973) 57-60

[15] N E Steenrod, Cohomology operations, Annals of Mathematics Studies 50, Princeton Univ. Press (1962) MR0145525

[16] R E Stong, Notes on cobordism theory, Mathematical Notes 7, Princeton Univ. Press (1968) MR0248858

[17] A Wilfong, Toric polynomial generators of complex cobordism, Algebr. Geom. Topol. 16 (2016) 1473-1491 MR3523047

ZL: School of Mathematical Sciences, Fudan University

Shanghai, 200433, China

TP: Department of Mathematics and Mechanics, Moscow State University

Leninskie Gory, Moscow, 119991, Russia

TP: Institute for Theoretical and Experimental Physics

Main Cheremushkinskaya St, Moscow 117218, Russia

TP: Institute for Information Transmission Problems, Russian Academy of Sciences

Bolshoy Karetny Lane, Moscow 127051, Russia 
zlu@fudan.edu.cn, tpanov@mech.math.msu.su

http://homepage.fudan.edu.cn/zhilu/,

http://higeom.math.msu.su/people/taras/

Received: 2 June 2015 Revised: 15 October 2015 
